\title{
Efficacy and safety of imiquimod for verruca planae: A systematic review
}

\author{
Xin-rui Zhang", Bi-huan Xiao", Rui-qun Qi*, and Xing-hua Gao* \\ Department of Dermatology, No 1 Hospital of China Medical University, Shenyang 110001, PR China \\ \#These authors contributed equally to this work
}

\begin{abstract}
Objective: To assess the efficacy and safety of imiquimod for treating verruca planae.

Methods: We searched the Pubmed, Cochrane Register of Controlled Trials, EMbase, CBM, CNKI and Wanfang databases (Chinese) to collect randomized controlled trials (RCTs). We screened the retrieved studies according to the predefined inclusion and exclusion criteria, evaluated the quality of include studies, and performed meta-analyses using the Cochrane Collaboration's RevMan 5.1. Software.

Results: Twenty-six RCTs involving 2169 patients with verruca planae were included and assessed. At the end of the 6th and $\geq 8$ th week, the effective rate of topical imiquimod was obviously higher than that of control [ $\mathrm{RR}=1.42,95 \% \mathrm{CI}(1.27,1.60), \mathrm{P}<0.00001 ; \mathrm{RR}=1.43,95 \% \mathrm{CI}(1.22,1.67)$, $\mathrm{P}<0.00001]$. The effective rate of imiquimod cream was higher than tretinoin cream, tazarotene gel and other antiviral drugs. $[\mathrm{RR}=1.41,95 \% \mathrm{CI}(1.25,1.59), \mathrm{P}<0.00001 ; \mathrm{RR}=1.76,95 \% \mathrm{CI}(1.48,2.10)$, $\mathrm{P}<0.00001 ; \mathrm{RR}=1.71,95 \% \mathrm{CI}(1.29,2.26), \mathrm{P}=0.0002]$. However, the effectiverate of imiquimod cream was lower than 5-ALA-PDT (RR=0.6, 95\%CI (0.5, 0.71), $\mathrm{P}<0.00001)$.

Conclusions: The limited evidence demonstrates that topical imiquimod is safe and efficient. More multiple central RCTs with large samples are required to verify these conclusions.
\end{abstract}

\section{Introduction}

Verruca planae are warts caused by the human papilloma virus (HPV), mostly by HPV type3, 10, 28 and 41 . They primarily affect children and young adults [1]. The treatment cycle is usually prolonged. Current treatment options are anti-viral, immune-modulation and destruction of the infected tissue.

Imiquimod is a low molecular weight, synthetic immune response modifier which does not exert its antiviral effects directly on virusinfected cells. Imiquimod induces the production of antiviral cytokines that enhance cellular immunity necessary for the control or elimination of HPV infection. Imiquimod is often prescribed for condyloma acuminatum [2]. In recent years, there are reports that it has been used for flat warts and molluscum contagiosum, with satisfactory curative effect [3]. Here we performed a systematic review on the efficacy and safety of imiquimod cream in the treatment of verruca planae in order to provide the evidence for clinical decision.

\section{Materials and methods}

\section{Eligibility criteria}

Inclusion and exclusion criteria were determined before the search was conducted. We included studies comparing the efficacy and safety of Imiquimod with other drugs on patients with verruca planae. Included studies must be randomized controlled trial (RCT). Case reports, reviews and document of incomplete data were excluded.

\section{Intervention measure}

The test group for $5 \%$ imiquimod cream alone or in combination with other treatment methods.

\section{Outcome indicator}

Cured: skin lesions completely disappeared or decreased $>90 \%$. Significantly improved: skin lesions decreased $>60 \%$ (or $>70 \%$ ); Improved: over $30 \%$, but less than $70 \%$ of decrease of skin lesions; Invalid: less than $30 \%$ in decrease of kin lesions. The effective rate is the sum of cured and significantly improved by the total number of the tested patients.

\section{Data sources and searches}

To identify relevant studies, three reviewers (Z.X.R, X.B.H, H.X.J) systematically searched MEDLINE, Cochrane Central Register of Controlled Trials, Embase, China National Knowledge Infrastructure database (CNKI), China Biology Medicine disc (CBM) and Wanfang Data Knowledge Service Platform. Search terms were based on specific agents ("Imiquimod," "Verruca Planae," "Plane Warts," "Flat Warts,"

Correspondence to: Rui-qun Qi, MD, PhD, Department of Dermatology, No 1 Hospital of China Medical University, Shenyang 110001, China, Tel: +86248328 2742; Fax: +86 248328 2742; E-mail: xiaoqiliumin@163.com

Xing-hua Gao, MD, PhD, Department of Dermatology, No 1 Hospital of China Medical University, Shenyang 110001, China, Tel: +86 248328 2326; Fax: +86 24 8328 2326; E-mail: gaobarry@hotmail.com

Key words: imiquimod, verruca planae, plane warts, flat warts, systematic review, randomized controlled trial

Received: July 01, 2016; Accepted: August 03, 2016; Published: August 05, 2016 
"Systematic review," "Randomized controlled trial," “Treatment").

\section{Study selection}

To determine eligibility for inclusion in the review, we searched all titles and abstracts that analyses the efficacy of imiquimod for treating verruca planae with control group. There were no limitations on the study design, participant's age, gender, or nationality. We identified 68 articles in the initial search. Two of them were in English, others in Chinese. Through manual review of the citations from these articles, we removed 39 articles including general reviews, case reports, investigative research, commentaries and other non-clinical trials. We identified 28 original studies that were eligible for inclusion criteria assessment. After reviewing the full text of these 28 studies, we excluded 2 articles of non RCT nature. In the end, we selected 26 studies that met the inclusion criteria for this systematic review [4-29]. The search processes are presented in chart in Figure 1.

\section{Data extraction}

Studies were selected independently by two researchers who used the same criteria. The third researcher was involved in the discussion if there were controversial issues. If the information is not complete or not clear, we contact original author to obtain the information and determine whether the study will be included.

\section{Quality assessment}

We used RevMan 5.1 (Review Manager Version 5.1) to analyze the quality of the data, including random sequence generation, allocation concealment, blinding of participants and personnel, blinding of outcome assessment, incomplete outcome data, selective reporting and other bias (Figure 2).

\section{Data synthesis and analysis}

We analysed the data using RevMan 5.1.Chi square test was used to test for heterogeneity. The degree of heterogeneity between studies was assessed using the $\mathrm{I}^{2}$ test. And $\mathrm{I}^{2}$ value $>50 \%$ was considered there existed substantial heterogeneity. In such a case, random effects model was used. Results were expressed with relative risk (RR) and $95 \%$ confidence interval (CI) and the test level $\alpha=0.05$. Publication bias was displayed graphically by using funnel plots (Figures 1 and 2).

\section{Result}

\section{Characteristics of the included publications}

The basic characteristics of the included studies were shown in Table 1, including the total number of cases, the number of excluded and the lost, the age, course of disease, the number of effective cases and the intervention measures.

\section{Quality assessment}

All included studies were referred to the randomized grouping, and only 4 studies $[4,9,10,20]$ described the details. All the studies were unclear in reporting allocation concealment and blinding. 5 studies $[5,7,8,14,29]$ reported the number of withdraw. Assessment of methodological quality of included studies was presented in Figure 2.

\section{Therapeutic evaluation}

Total effect: Twenty six RCTs involving 2169 patients with verruca planae were included and assessed. Results showed that the effective rate of topical imiquimod combined with intramuscular injection or other oral drugs in the treatment of flat wart was obviously higher than using other drugs alone. ( $\mathrm{RR}=1.35$ (95\% CI 1.181.54.), $\mathrm{P}<0.00001)$ (Figure 3), 10 studies [4,10-11,17-18,20-21,23,27,29] described the efficacy at the end of 4 weeks. As there exist statistical heterogeneity among the studies $\left(\mathrm{P}<0.00001, \mathrm{I}^{2}=82 \%\right)$, meta-analysis was performed using a random effects model. The results showed that the efficacy of test group was significantly higher than that in control group. The difference had no statistical significance. $(\mathrm{RR}=1.81,95 \% \mathrm{CI}(0.91,1.54), \mathrm{P}=0.21)$ (Figure 4A.2.1.1.). 6 studies $[5,17-18,20-22]$ described the efficacy at the end

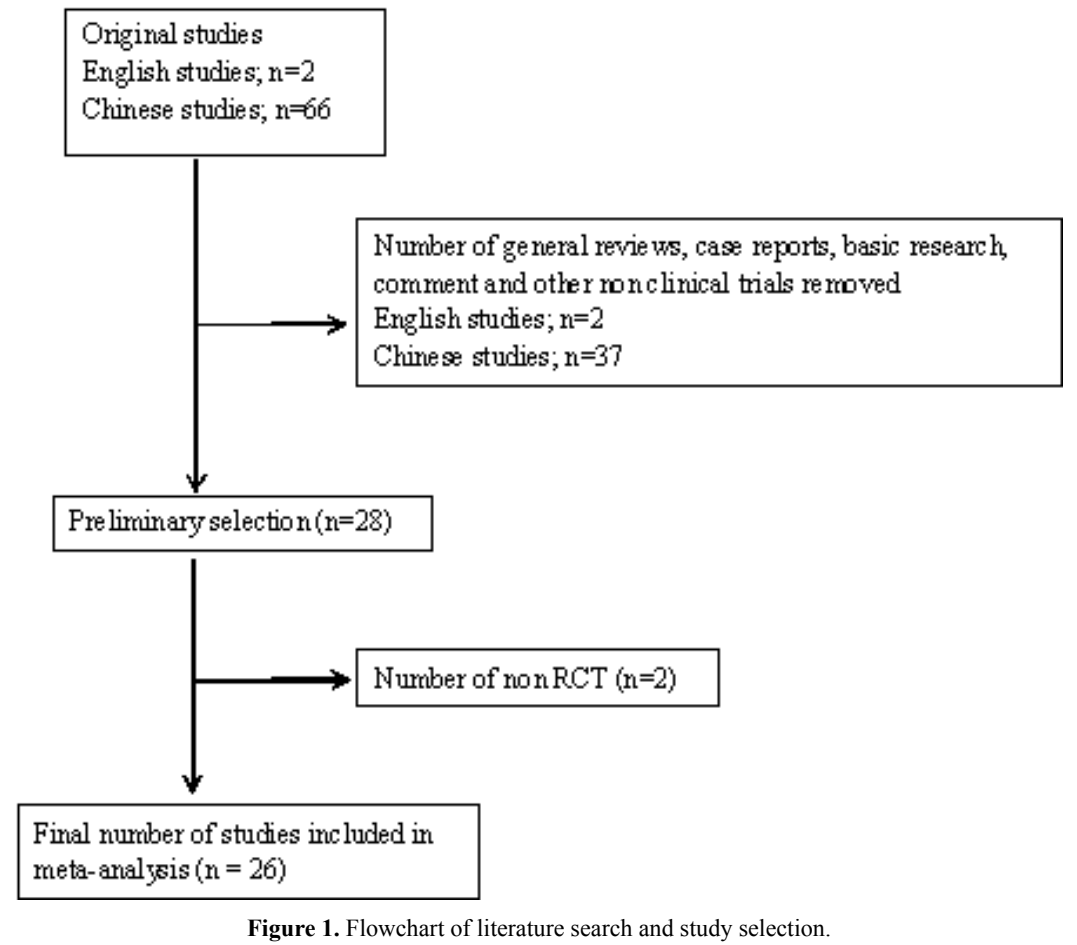


A

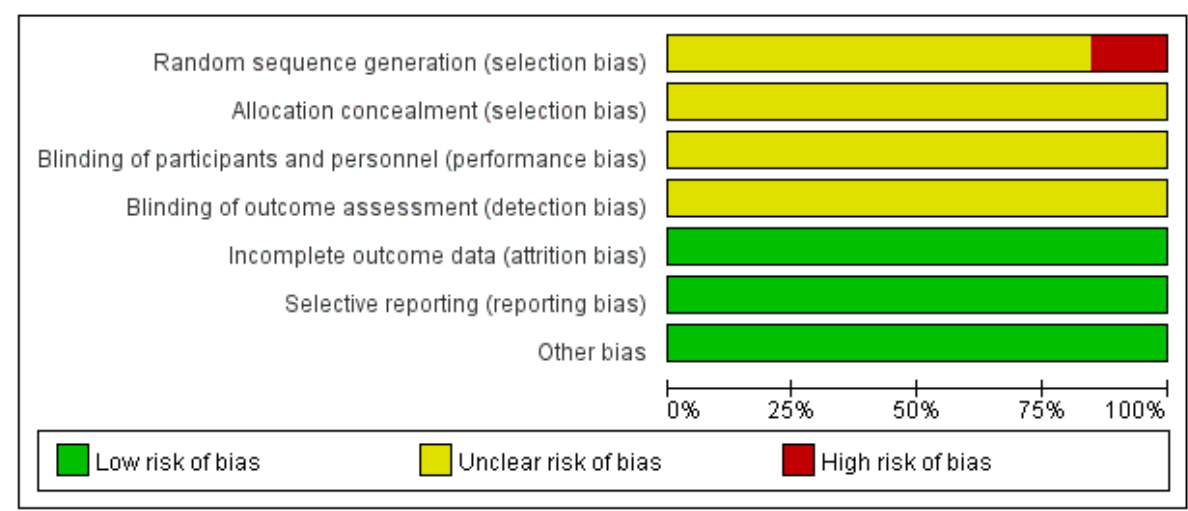

B

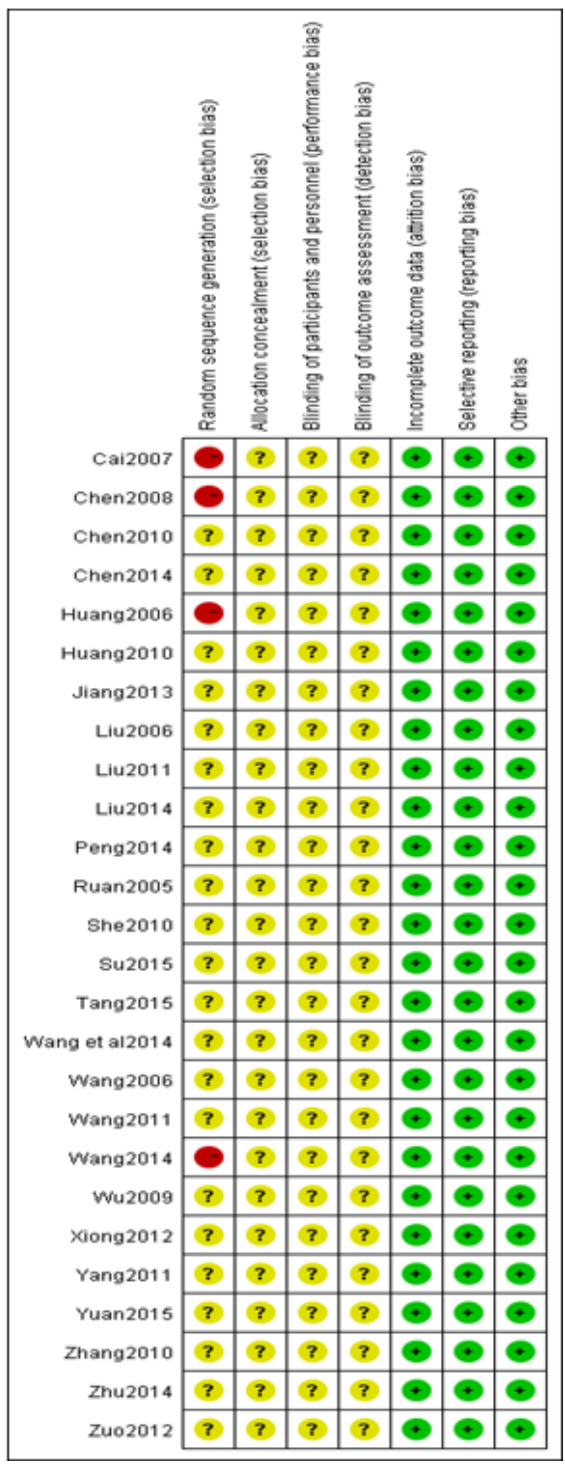

Figure 2. Assessment of methodological quality of included studies (2A.Risk of bias graph; 2B. Risk of bias summary).

of 6 weeks. As there was no statistical heterogeneity $\left(\mathrm{P}=0.08, \mathrm{I}^{2}=48 \%\right)$, meta-analysis was performed using a fixed effects model. The results showed that the efficacy in the test group was significantly higher than that in control group $(\mathrm{RR}=1.42,95 \% \mathrm{CI}(1.27,1.60), \mathrm{P}<0.00001)$ (Figure 4B), 19 studies [4,6-21,24-26,28] described the efficacy at the end of 8 weeks. As there existed statistical heterogeneity $\left(\mathrm{P}<0.00001, \mathrm{I}^{2}=80 \%\right)$, meta-analysis was performed using a random effects model. The results showed that the efficacy in the test group was significantly higher than that in control group $(\mathrm{RR}=1.43,95 \% \mathrm{CI}(1.22,1.67), \mathrm{P}<0.00001$ ] (Figure 4A.2.1.2.).

Imiquimod cream $v s$. Tretinoin cream: A total of 8 studies [4-11] were included, of which 3 studies [4-6] of the test group is imiquimod cream combined with BCG -PSN, the control group is tretinoin cream combined with BCG-PSN, 3 studies [7-9] of the test group was imiquimod cream only, the control group was tretinoin cream only. The 2 studies $[10,11]$ of test group was imiquimod cream combined with tretinoin cream once a day each, the control group was only tretinoin cream once per day; results showed that the effective rate in imiquimod group was significantly higher than tretinoin cream group $(\mathrm{RR}=1.41,95 \% \mathrm{CI}(1.25,1.59), \mathrm{P}<0.00001)$; the effective rate of imiquimod combined with BCG-PSN group was higher than that of tretinoin cream combined with BCG-PSN group $(\mathrm{RR}=1.56$, 95\% CI $(1.27,1.91), \mathrm{P}<0.0001)$; the effective rate of imiquimod alone or in combination with tretinoin cream group was higher than that of tretinoin cream alone group $(\mathrm{RR}=1.32,95 \% \mathrm{CI}(1.14,1.54), \mathrm{P}=0.0003)$ (Figure 5A.3.1.1.).

Imiquimod cream vs. Tazarotene gel: A total of 4 studies [1215] were included, of which the test group was imiquimod cream combined with tazarotene gel once a day each, the control group was tazarotene gel alone once per day. The results showed the effective rate in test group was higher than tazarotene group $(\mathrm{RR}=1.76,95 \% \mathrm{CI}(1.48$, 2.10), $\mathrm{P}<0.00001$ ) (Figure 5A.3.1.2.). One study [16] reported that the effective rate in imiquimod group was higher than that of adapalene group. 
Table 1. Characteristics of included studies.

\begin{tabular}{|c|c|c|c|c|c|c|c|c|c|c|}
\hline \multirow{2}{*}{$\begin{array}{l}\text { First author; } \\
\text { publication year }\end{array}$} & \multirow{2}{*}{\begin{tabular}{|l|} 
Age (year) \\
$\mathrm{T}$ \\
\end{tabular}} & \multirow{2}{*}{ C } & \multicolumn{2}{|c|}{ Course of disease } & \multirow{2}{*}{\begin{tabular}{|l|} 
Exit number \\
$(\mathrm{T} / \mathrm{C})$ \\
\end{tabular}} & \multirow{2}{*}{\begin{tabular}{|l|} 
Total number \\
$(\mathrm{T} / \mathrm{C})$ \\
\end{tabular}} & \multirow{2}{*}{$\begin{array}{l}\text { Effective } \\
(\mathrm{T} / \mathrm{C})\end{array}$} & \multirow{2}{*}{$\begin{array}{l}\text { course of } \\
\text { treatment }\end{array}$} & \multicolumn{2}{|c|}{ Intervention measures } \\
\hline & & & $\mathrm{T}$ & $\mathrm{C}$ & & & & & $\mathrm{T}$ & $\mathrm{C}$ \\
\hline \multirow[t]{3}{*}{ Wang (2014) } & $19-39$ & $20-40$ & $3 m-2 y$ & $2 m-2 y$ & - & $40 / 40$ & $1 \mathrm{~m}: 17 / 15$ & $1 \mathrm{~m}, 2 \mathrm{~m}, 3 \mathrm{~m}$ & $\begin{array}{l}5 \% \text { imiquimod } \\
\text { cream, } 1 \text { time per } \\
\text { day, external use } \\
\text { and BCG-PSN, } 3 \\
\text { times per week, } \\
2 \text { ml each time, } \\
\text { intramuscular } \\
\text { injection }\end{array}$ & $\begin{array}{l}0.1 \% \text { vitamin } \mathrm{A} \text { acid cream, } \\
1 \text { time per day and BCG- } \\
\text { PSN, } 3 \text { times per week, } 2 \\
\text { ml each time, intramuscular } \\
\text { injection }\end{array}$ \\
\hline & & & & & & & $2 \mathrm{~m}: 30 / 18$ & & & \\
\hline & & & & & & & $3 \mathrm{~m}: 34 / 21$ & & & \\
\hline Liu (2014) & $12-34$ & & $7 \mathrm{~m}-3.5 \mathrm{y}$ & & 3 & $37 / 37$ & $24 / 14$ & $6 w$ & $\begin{array}{l}5 \% \text { imiquimod } \\
\text { cream, } 1 \text { time per } \\
\text { day, external use } \\
\text { and BCG-PSN, } \\
\text { every other day, } \\
\text { intramuscular } \\
\text { injection }\end{array}$ & $\begin{array}{l}0.1 \% \text { vitamin A acid cream, } \\
1 \text { time per day, external use } \\
\text { and BCG-PSN, every other } \\
\text { day, intramuscular injection }\end{array}$ \\
\hline Huang (2010) & $8-40$ & & $1 w-2 y$ & & - & $40 / 38$ & $34 / 21$ & $8 w$ & $\begin{array}{l}5 \% \text { imiquimod } \\
\text { cream, } 1 \text { time per } \\
\text { day, external use } \\
\text { and BCG-PSN, } 3 \\
\text { times per week, } \\
2 \text { ml each time, } \\
\text { intramuscular } \\
\text { injection }\end{array}$ & $\begin{array}{l}0.1 \% \text { vitamin A acid cream, } \\
1 \text { time per day, external } \\
\text { use and BCG-PSN, } 3 \text { times } \\
\text { per week, } 2 \mathrm{ml} \text { each time, } \\
\text { intramuscular injection }\end{array}$ \\
\hline Ruan (2005) & $10-45$ & & $1 \mathrm{~m}-51 \mathrm{~m}$ & & 4 & $34 / 33$ & $26 / 18$ & $8 w$ & \begin{tabular}{|l|}
$5 \%$ imiquimod \\
cream and $0.025 \%$ \\
vitamin A acid \\
cream, every other \\
day, alternative \\
external use
\end{tabular} & $\begin{array}{l}0.025 \% \text { vitamin } A \text { acid } \\
\text { cream, } 1 \text { time per day, } \\
\text { external use }\end{array}$ \\
\hline Zuo (2012) & $10-43$ & & $1 \mathrm{~m}-5 \mathrm{y}$ & & 5 & $35 / 33$ & $29 / 17$ & $8 w$ & $\begin{array}{l}5 \% \text { imiquimod } \\
\text { cream and } 0.025 \% \\
\text { vitamin A acid } \\
\text { cream, every other } \\
\text { day, alternative } \\
\text { external use }\end{array}$ & $\begin{array}{l}0.025 \% \text { vitamin A acid } \\
\text { cream, } 1 \text { time per day, } \\
\text { external use }\end{array}$ \\
\hline Chen (2008) & $6-41$ & & $3 w-4 y$ & & - & $23 / 22$ & $13 / 10$ & $8 w$ & $\begin{array}{l}5 \% \text { imiquimod } \\
\text { cream, every other } \\
\text { day, external use }\end{array}$ & $\begin{array}{l}0.1 \% \text { vitamin A acid cream, } \\
1 \text { time per day, external use }\end{array}$ \\
\hline \multirow[t]{2}{*}{ Huang (2006) } & $12-51$ & & $2 w-2.5 y$ & & - & $32 / 26$ & $4 w: 25 / 16$ & $8 w$ & $\begin{array}{l}5 \% \text { imiquimod } \\
\text { cream, every other } \\
\text { day, external use }\end{array}$ & $\begin{array}{l}0.05 \% \text { vitamin A acid } \\
\text { cream, } 1 \text { time per day, } \\
\text { external use }\end{array}$ \\
\hline & & & & & & & $8 w: 26 / 16$ & & & \\
\hline Wang (2006) & $15-46$ & & $2 w-2 y$ & & - & $60 / 58$ & $45 / 32$ & $4 w$ & $\begin{array}{l}5 \% \text { imiquimod } \\
\text { cream, every other } \\
\text { day, external use }\end{array}$ & $\begin{array}{l}0.1 \% \text { vitamin A acid cream, } \\
1 \text { time per day, external use }\end{array}$ \\
\hline Chen (2014) & $15-34$ & $14-35$ & $\begin{array}{l}\text { half month-36m } \\
1 \mathrm{~m}-36 \mathrm{~m}\end{array}$ & & - & $45 / 37$ & $32 / 16$ & $8 w$ & \begin{tabular}{|l|}
$5 \%$ imiquimod \\
cream and \\
tazarotene gel, \\
every other day, \\
alternative external \\
use
\end{tabular} & $\begin{array}{l}0.1 \% \text { tazarotene gel, } 1 \text { time } \\
\text { per day, external use }\end{array}$ \\
\hline Xiong (2012) & $10-42$ & & $6 w-3 y$ & & - & $46 / 48$ & $42 / 25$ & $8 w$ & $\begin{array}{l}\text { imiquimod cream } \\
\text { and tazarotene gel, } \\
\text { every other day, } \\
\text { alternative external } \\
\text { use }\end{array}$ & $\begin{array}{l}\text { tazarotene gel, } 1 \text { time per } \\
\text { day, external use }\end{array}$ \\
\hline Yang (2011) & 29.9 & & $6 m-15 y$ & & 3 & $34 / 31$ & $26 / 17$ & $8 w$ & \begin{tabular}{|l|}
$5 \%$ imiquimod \\
cream and \\
tazarotene gel, \\
every other day, \\
alternative external \\
use
\end{tabular} & $\begin{array}{l}0.05 \% \text { tazarotene gel, } 1 \\
\text { time per day, external use }\end{array}$ \\
\hline Zhang (2010) & $12-45$ & & $1 \mathrm{~m}-6 \mathrm{y}$ & & - & $47 / 81$ & $33 / 24$ & $8 w$ & $\begin{array}{l}\text { imiquimod cream } \\
\text { and tazarotene gel, } \\
\text { every other day, } \\
\text { alternative external } \\
\text { use }\end{array}$ & $\begin{array}{l}\text { tazarotene gel, } 1 \text { time per } \\
\text { day, external use }\end{array}$ \\
\hline
\end{tabular}




\begin{tabular}{|c|c|c|c|c|c|c|c|c|c|c|}
\hline Yuan (2015) & $13-51$ & & $2 m-4 y$ & & - & $52 / 42$ & $41 / 23$ & $16 \mathrm{w}$ & \begin{tabular}{|l|}
$5 \%$ imiquimod \\
cream and \\
adapalene gel \\
every other day, \\
alternative external \\
use
\end{tabular} & $\begin{array}{l}\text { adapalene gel, } 1 \text { time per } \\
\text { day, external use }\end{array}$ \\
\hline \multirow[t]{3}{*}{ Peng (2014) } & $12-42$ & $15-45$ & $1 \mathrm{~m}-2 \mathrm{y}$ & $15 \mathrm{~d}-3 \mathrm{~m}$ & & $110 / 120$ & $2 \mathrm{w}: 66 / 55$ & $2 \mathrm{w}, 4 \mathrm{w}, 6 \mathrm{w}$ & $\begin{array}{l}5 \% \text { imiquimod } \\
\text { cream and } \\
\text { adapalene gel, } \\
1 \text { time per day, } \\
\text { external use }\end{array}$ & $\begin{array}{l}\text { adapalene gel, } 1 \text { time per } \\
\text { day, external use }\end{array}$ \\
\hline & & & & & & & 4w:77/51 & & & \\
\hline & & & & & & & $6 \mathrm{w}: 97 / 80$ & & & \\
\hline \multirow[t]{4}{*}{ Chen (2010) } & $11-42$ & & $9 \mathrm{~m}-6 \mathrm{y}$ & & - & $59 / 57$ & 2w:9/5 & $2 \mathrm{w}, 4 \mathrm{w}, 6 \mathrm{w}, 8 \mathrm{w}$ & $\begin{array}{l}5 \% \text { imiquimod } \\
\text { cream, } 1 \text { time per } \\
\text { day, external use } \\
\text { and BCG-PSN, } \\
\text { every other day, } \\
1 \mathrm{ml} \text { each time, } \\
\text { intramuscular } \\
\text { injection }\end{array}$ & $\begin{array}{l}\text { matrix, every other day, } \\
\text { external use and BCG- } \\
\text { PSN, every other day, } 1 \mathrm{ml} \\
\text { each time, intramuscular } \\
\text { injection }\end{array}$ \\
\hline & & & & & & & $4 w 39 / 25$ & & & \\
\hline & & & & & & & $6 \mathrm{w}: 51 / 37$ & & & \\
\hline & & & & & & & $8 w: 56 / 42$ & & & \\
\hline She (2010) & $18-47$ & & $1 \mathrm{~m}-4 \mathrm{y}$ & & - & $34 / 34$ & $18 / 14$ & $8 w$ & $\begin{array}{l}5 \% \text { imiquimod } \\
\text { cream, } 1 \text { time per } \\
\text { day, external use } \\
\text { and famciclovir } \\
\text { tablets, } 2 \text { times } \\
\text { per day, } 0.2 \mathrm{~g} \text {, oral } \\
\text { administration }\end{array}$ & $\begin{array}{l}\text { famciclovir tablets, } 2 \\
\text { times per day, } 0.2 \mathrm{~g} \text {, oral } \\
\text { administration }\end{array}$ \\
\hline \multirow[t]{4}{*}{ Cai (2007) } & $7-40$ & & $\begin{array}{l}\text { half of month- } \\
24 \mathrm{~m}\end{array}$ & & - & $32 / 31$ & $2 w: 2 / 2$ & $2 w, 4 w, 6 w, 8 w$ & $\begin{array}{l}5 \% \text { imiquimod } \\
\text { cream, every other } \\
\text { day, external use }\end{array}$ & $\begin{array}{l}\text { placebo, every other day, } \\
\text { external use }\end{array}$ \\
\hline & & & & & & & $4 w: 4 / 2$ & & & \\
\hline & & & & & & & $6 \mathrm{w}: 6 / 2$ & & & \\
\hline & & & & & & & $8 w: 10 / 3$ & & & \\
\hline \multirow[t]{4}{*}{ Wang (2011) } & $17-38$ & $18-40$ & $2 m-3 y$ & $3 m-4 y$ & - & $45 / 40$ & $2 \mathrm{w}: 6 / 3$ & $2 \mathrm{w}, 4 \mathrm{w}, 6 \mathrm{w}, 8 \mathrm{w}$ & $\begin{array}{l}5 \% \text { imiquimod } \\
\text { cream, } 1 \text { time } \\
\text { per day, external } \\
\text { use and transfer } \\
\text { factor, } 3 \mathrm{mg}, \\
\text { twice per week, } \\
\text { subcutaneous } \\
\text { injection }\end{array}$ & $\begin{array}{l}\text { transfer factor, } 3 \mathrm{mg} \text {, twice } \\
\text { per week, subcutaneous } \\
\text { injection }\end{array}$ \\
\hline & & & & & & & $4 w: 16 / 9$ & & & \\
\hline & & & & & & & $6 \mathrm{w}: 25 / 12$ & & & \\
\hline & & & & & & & $8 w: 35 / 16$ & & & \\
\hline Zhu (2014) & - & & $3 \mathrm{~m}-8 \mathrm{y}$ & & - & $30 / 30$ & $23 / 10$ & $6 \mathrm{w}$ & $\begin{array}{l}5 \% \text { imiquimod } \\
\text { cream, 1 time } \\
\text { per day, external } \\
\text { use and placenta } \\
\text { polypeptide } \\
\text { injection, } 1 \\
\text { time per day, } \\
\text { intramuscular } \\
\text { injection }\end{array}$ & $\begin{array}{l}\text { placenta polypeptide } \\
\text { injection, } 1 \text { time per day, } \\
\text { intramuscular injection }\end{array}$ \\
\hline Liu (2011) & $8-42$ & & $20 \mathrm{~d}-2 \mathrm{y}$ & & - & $40 / 39$ & $40 / 26$ & $4 w$ & $\begin{array}{l}5 \% \text { imiquimod } \\
\text { cream, every } \\
\text { other day, external } \\
\text { use and IFN-a } 2 \mathrm{~b} \\
\text { cream, } 3 \text { times per } \\
\text { day, external use }\end{array}$ & $\begin{array}{l}\text { IFN-a2b cream, } 3 \text { times per } \\
\text { day, external use }\end{array}$ \\
\hline Tang (2015) & $14-51$ & $16-50$ & - & & - & $41 / 40$ & $41 / 37$ & $8 w$ & $\begin{array}{l}\text { imiquimod cream, } \\
\text { every other day, } \\
\text { external use and } \\
\text { ALA-PDT, } 1 \text { time } \\
\text { per } 7 \text { to } 10 \text { days }\end{array}$ & $\begin{array}{l}\text { ALA-PDT, } 1 \text { time per } 7 \\
\text { to10 days }\end{array}$ \\
\hline Liu (2006) & $13-36$ & $13-40$ & $4 m-3 y$ & $3 m-4 y$ & - & $45 / 45$ & $29 / 15$ & $8 w$ & $\begin{array}{l}5 \% \text { imiquimod } \\
\text { cream, every other } \\
\text { day, external use }\end{array}$ & $\begin{array}{l}\text { Ftibamzone liniment, } 2 \\
\text { times per day, external use }\end{array}$ \\
\hline
\end{tabular}




\begin{tabular}{|c|c|c|c|c|c|c|c|c|c|c|}
\hline Wu (2009) & $12-50$ & & $2 w-6 y$ & & - & $35 / 32$ & $30 / 18$ & $8 w$ & \begin{tabular}{|l|}
$5 \%$ imiquimod \\
cream, 1 time per \\
day, external use \\
and Pidotimod \\
powder, two \\
copies per day, \\
oral administration
\end{tabular} & $\begin{array}{l}\text { Penciclovir cream, } 1 \\
\text { time per day, external use } \\
\text { and Pidotimod powder, } \\
\text { two copies per day, oral } \\
\text { administration }\end{array}$ \\
\hline Wang (2014) & $18-26$ & $19-25$ & $1 y-3 y$ & $1 y-3 y$ & - & $35 / 35$ & $20 / 35$ & $4 w$ & $\begin{array}{l}5 \% \text { imiquimod } \\
\text { cream, every other } \\
\text { day, external use } \\
\end{array}$ & ALA-PDT, once a week \\
\hline $\mathrm{Su}(2015)$ & & & - & & - & $38 / 40$ & $22 / 40$ & $8 w$ & $\begin{array}{l}5 \% \text { imiquimod } \\
\text { cream, every other } \\
\text { day, external use }\end{array}$ & $\begin{array}{l}\text { ALA-PDT, } 1 \text { time per } 10 \text { to } \\
14 \text { days }\end{array}$ \\
\hline Jiang (2013) & $18-26$ & $19-25$ & $1 y-3 y$ & $1 y-3 y$ & 1 & $15 / 16$ & $10 / 16$ & $3 w$ & $\begin{array}{l}5 \% \text { imiquimod } \\
\text { cream, every other } \\
\text { day, external use }\end{array}$ & ALA-PDT, once a week \\
\hline
\end{tabular}

\section{Experimental Control Risk Ratio}

Studv or Subgroup

\subsubsection{Total Effect}

\begin{tabular}{|c|c|c|c|c|c|}
\hline Cai2007 & 22 & 32 & 9 & 31 & $2.5 \%$ \\
\hline Chen2008 & 13 & 23 & 10 & 22 & $2.6 \%$ \\
\hline Chen2010 & 56 & 59 & 42 & 57 & $4.9 \%$ \\
\hline Chen2014 & 32 & 45 & 16 & 37 & $3.5 \%$ \\
\hline Huang2006 & 26 & 32 & 16 & 26 & $3.9 \%$ \\
\hline Huang2010 & 34 & 40 & 21 & 38 & $4.1 \%$ \\
\hline Jiang2013 & 10 & 15 & 16 & 16 & $3.8 \%$ \\
\hline Liu2006 & 29 & 45 & 15 & 45 & $3.2 \%$ \\
\hline Liu2011 & 40 & 40 & 26 & 39 & $4.6 \%$ \\
\hline Liu2014 & 24 & 37 & 16 & 37 & $3.3 \%$ \\
\hline Peng2014 & 97 & 110 & 80 & 120 & $5.0 \%$ \\
\hline Ruan2005 & 26 & 34 & 20 & 33 & $3.9 \%$ \\
\hline She2010 & 18 & 34 & 14 & 34 & $2.9 \%$ \\
\hline Sแ2015 & 22 & 38 & 40 & 40 & $4.3 \%$ \\
\hline Tang2015 & 41 & 41 & 37 & 40 & $5.1 \%$ \\
\hline Wang et al2014 & 20 & 35 & 35 & 35 & $4.2 \%$ \\
\hline Wang2006 & 45 & 60 & 32 & 58 & $4.3 \%$ \\
\hline Wang2011 & 35 & 45 & 16 & 40 & $3.5 \%$ \\
\hline Wang2014 & 34 & 40 & 21 & 40 & $4.0 \%$ \\
\hline Wu2009 & 30 & 35 & 18 & 32 & $3.9 \%$ \\
\hline Xiong2012 & 42 & 46 & 25 & 48 & $4.2 \%$ \\
\hline Yang2011 & 26 & 34 & 18 & 31 & $3.8 \%$ \\
\hline Yuan2015 & 41 & 52 & 23 & 42 & $4.1 \%$ \\
\hline Zhang2010 & 33 & 47 & 24 & 81 & $3.6 \%$ \\
\hline Zhu2014 & 23 & 30 & 10 & 30 & $2.8 \%$ \\
\hline Zuo2012 & 29 & 35 & 20 & 33 & $4.1 \%$ \\
\hline Subtotal $(95 \% \mathrm{Cl})$ & & 1084 & & 1085 & $100.0 \%$ \\
\hline Total events & 848 & & 620 & & \\
\hline
\end{tabular}

Heterogeneity: Tau $^{2}=0.08 ; \mathrm{Ch}^{2}=133.93, \mathrm{df}=25(\mathrm{P}=0.00001) ; \mathrm{F}^{2}=81 \%$

Test for overall effect: $Z=4.50$ ( $P=0.00001$ )

Total $(95 \% \mathrm{Cl})$

1084 848

$1085100.0 \%$ 620

Heterogeneity: $\mathrm{Tau}^{2}=0.08 ; \mathrm{Chi}^{2}=133.93, \mathrm{df}=25(\mathrm{P}=0.00001) ; \mathrm{I}^{2}=81 \%$

Test for overall effect: $Z=4.50$ ( $\mathrm{P}=0.00001$ )

Test for subaroun differences: Not anolicable

Figure 3. Forest plot for the total effect of imiquimod in the treatment of verruca planae.
Risk Ratio

M-H, Random, $95 \% \mathrm{Cl}$

$2.37[1.30,4.31]$

$1.24[0.70,2.22]$

$1.29[1.09,1.52]$

$1.64[1.09,2.49]$

$1.32[0.93,1.87]$

$1.54[1.12,2.11]$

$0.68[0.47,0.97]$

$1.93[1.21,3.08]$

$1.49[1.19,1.86]$

$1.50[0.97,2.33]$

$1.32[1.15,1.53]$

$1.26[0.91,1.76]$

$1.29[0.77,2.14]$

$0.58[0.45,0.77]$

$1.08[0.98,1.19]$

$0.58[0.43,0.77]$

$1.36[1.03,1.79]$

$1.94[1.29,2.93]$

$1.62[1.17,2.23]$

$1.52[1.09,2.13]$

$1.75[1.32,2.33]$

$1.32[0.93,1.87]$

$1.44[1.06,1.96]$

$2.37[1.61,3.48]$

$2.30[1.34,3.96]$

$1.37[1.00,1.87]$

$1.35[1.18,1.54]$ 


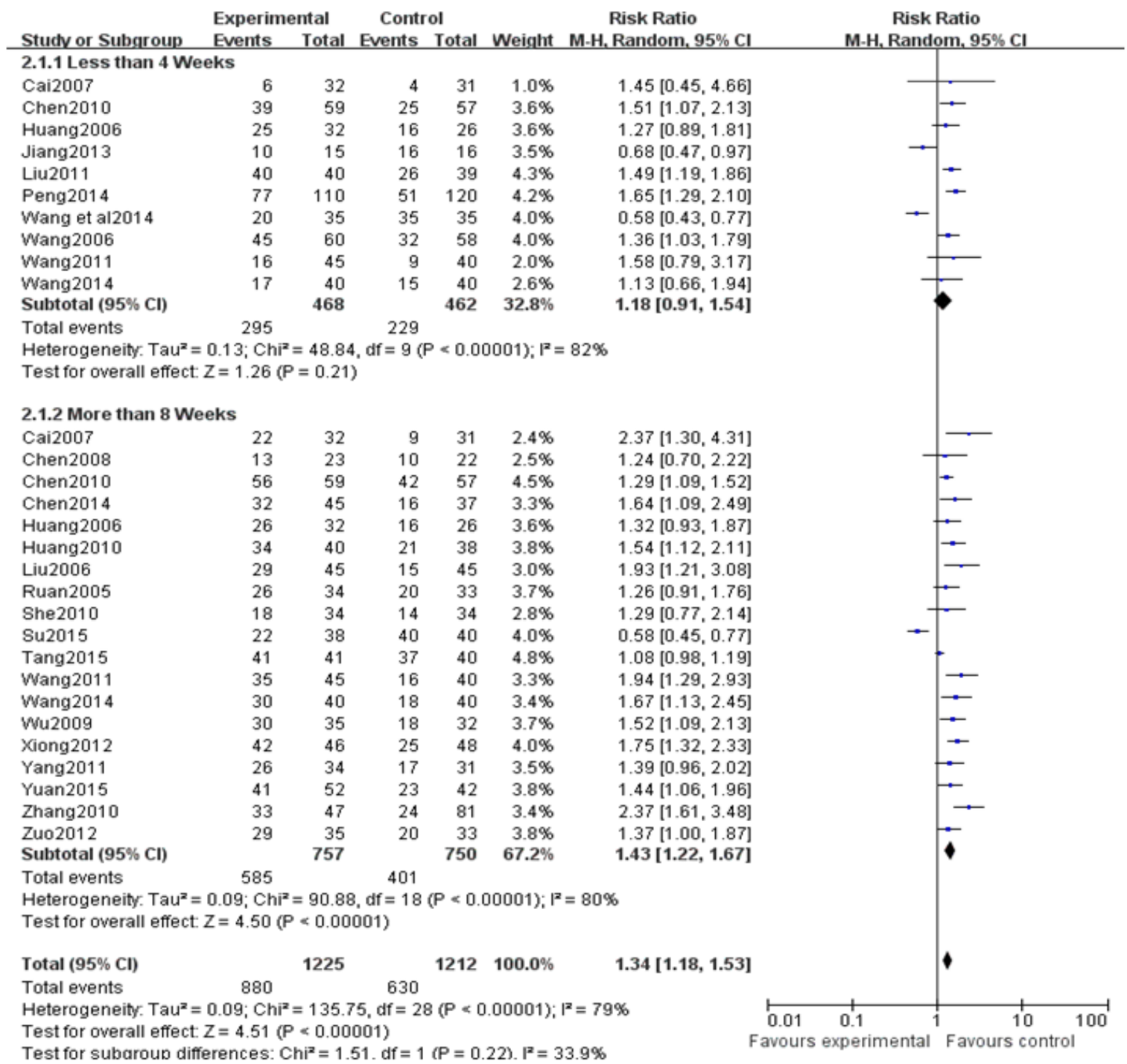

B

Experimental Control Risk Ratio

Rised

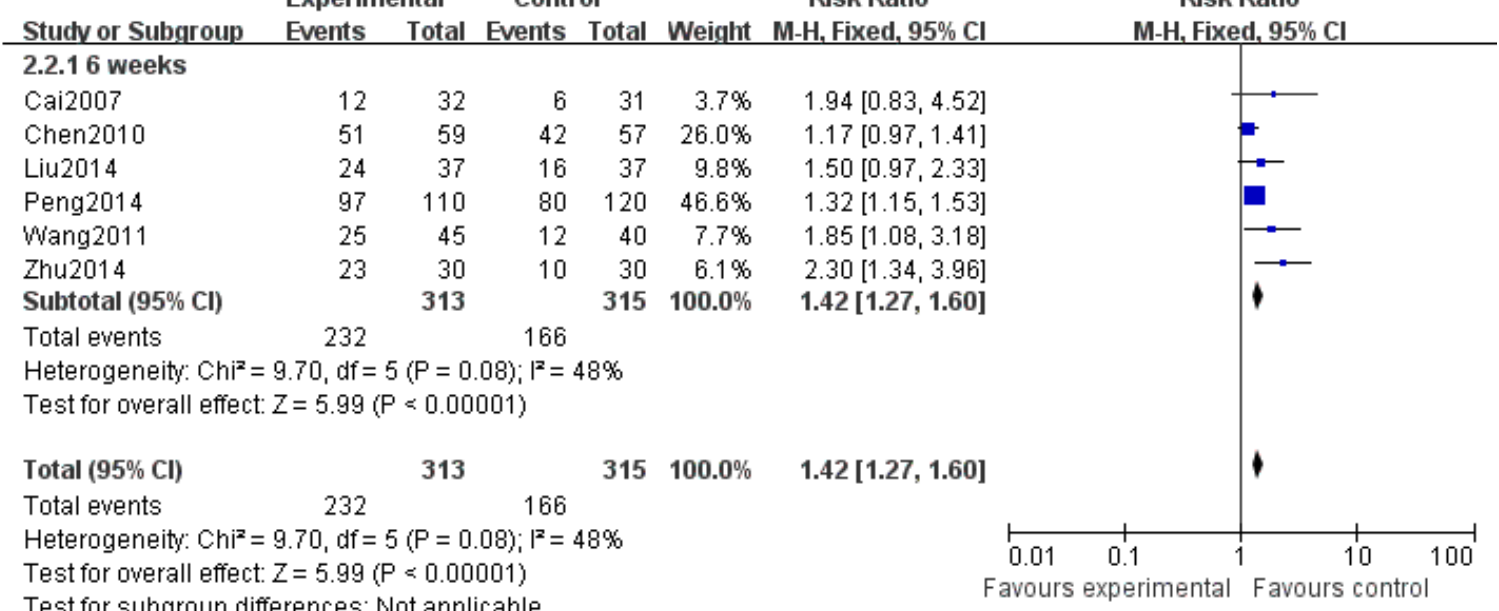

Test for subaroun differences: Not anplicable

Figure 4. Forest plot for treatment course (4A. 2.1.1. Efficacy in less than 4 weeks; 4A. 2.1.2. Efficacy in more than 8 weeks; 4B. Efficacy in 6 weeks). 
A

\begin{tabular}{|c|c|c|c|c|c|c|c|c|c|}
\hline \multirow[b]{2}{*}{ Studv or Subgroup } & \multicolumn{2}{|c|}{ Experimental } & \multicolumn{2}{|c|}{ Control } & \multirow{2}{*}{\multicolumn{2}{|c|}{$\begin{array}{c}\text { Risk Ratio } \\
\text { Weight } \quad M-H, \text { Fixed, } 95 \% \mathrm{CI}\end{array}$}} & \multirow{2}{*}{\multicolumn{2}{|c|}{$\begin{array}{l}\text { Risk Ratio } \\
\text { M-H, Fixed, } 95 \% \mathrm{Cl}\end{array}$}} & \\
\hline & Events & Total & Events & Total & & & & & \\
\hline \multicolumn{7}{|c|}{ 3.1.1 Imiquimod Cream vs Tretinoin Cream } & & & \\
\hline Chen 2008 & 13 & 23 & 10 & 22 & $2.8 \%$ & $1.24[0.70,2.22]$ & & - & \\
\hline Huang2006 & 26 & 32 & 16 & 26 & $4.9 \%$ & $1.32[0.93,1.87]$ & & + & \\
\hline Huang2010 & 34 & 40 & 21 & 38 & $5.9 \%$ & $1.54[1.12,2.11]$ & & $\rightarrow$ & \\
\hline Liu2014 & 24 & 37 & 16 & 37 & $4.4 \%$ & $1.50[0.97,2.33]$ & & 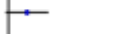 & \\
\hline Ruan2005 & 26 & 34 & 20 & 33 & $5.6 \%$ & $1.26[0.91,1.76]$ & & - & \\
\hline Wang2006 & 45 & 60 & 32 & 58 & $9.0 \%$ & $1.36[1.03,1.79]$ & & - & \\
\hline Wang2014 & 34 & 40 & 21 & 40 & $5.8 \%$ & $1.62[1.17,2.23]$ & & $\rightarrow$ & \\
\hline Zuo2012 & 29 & 35 & 20 & 33 & $5.7 \%$ & $1.37[1.00,1.87]$ & & - & \\
\hline Subtotal $(95 \% \mathrm{Cl})$ & & 301 & & 287 & $44.0 \%$ & $1.41[1.25,1.59]$ & & $\downarrow$ & \\
\hline Total events & 231 & & 156 & & & & & & \\
\hline \multicolumn{10}{|c|}{$\begin{array}{l}\text { Heterogeneity: } \mathrm{Chi}^{2}=1.93, \mathrm{df}=7(P=0.96) ; \mathrm{I}^{2}=0 \% \\
\text { Test for overall effect: } Z=5.51(P<0.00001)\end{array}$} \\
\hline \multicolumn{10}{|c|}{ 3.1.2 Imiquimod Cream vs Tazarotene Gel } \\
\hline Chen2014 & 32 & 45 & 16 & 37 & $4.8 \%$ & $1.64[1.09,2.49]$ & & $\rightarrow$ & \\
\hline Xiong2012 & 42 & 46 & 25 & 48 & $6.7 \%$ & $1.75[1.32,2.33]$ & & $\rightarrow$ & \\
\hline Yang2011 & 26 & 34 & 18 & 31 & $5.2 \%$ & $1.32[0.93,1.87]$ & & $\leftarrow$ & \\
\hline Zhang2010 & 33 & 47 & 24 & 81 & $4.9 \%$ & $2.37[1.61,3.48]$ & & $\overrightarrow{-}$ & \\
\hline Subtotal $(95 \% \mathrm{Cl})$ & & 172 & & 197 & $21.6 \%$ & $1.76[1.48,2.10]$ & & 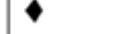 & \\
\hline Total events & 133 & & 83 & & & & & & \\
\hline \multicolumn{10}{|c|}{$\begin{array}{l}\text { Heterogeneity: } \mathrm{Chi}^{2}=5.02, \mathrm{df}=3(\mathrm{P}=0.17) ; \mathrm{I}^{2}=40 \% \\
\text { Test for overall effect: } \mathrm{Z}=6.29(\mathrm{P}<0.00001)\end{array}$} \\
\hline \multicolumn{10}{|c|}{ 3.1.3 Imiquimod Cream vs Other Antiviral Medications } \\
\hline Liu2006 & 29 & 45 & 15 & 45 & $4.1 \%$ & $1.93[1.21,3.08]$ & & $\rightarrow$ & \\
\hline Wu2009 & 30 & 35 & 18 & 32 & $5.2 \%$ & $1.52[1.09,2.13]$ & & $\rightarrow$ & \\
\hline Subtotal $(95 \% \mathrm{Cl})$ & & 80 & & 77 & $9.3 \%$ & $1.71[1.29,2.26]$ & & 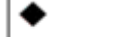 & \\
\hline Total events & 59 & & 33 & & & & & & \\
\hline \multicolumn{10}{|c|}{ Heterogeneity: $\mathrm{Chi}^{2}=0.71, \mathrm{df}=1(\mathrm{P}=0.40) ; \mathrm{I}^{2}=0 \%$} \\
\hline \multicolumn{10}{|c|}{ Test for overall effect: $Z=3.71(P=0.0002)$} \\
\hline \multicolumn{10}{|c|}{ 3.1.4 Imiquimod Cream vs ALA-PDT } \\
\hline Jiang2013 & 10 & 15 & 16 & 16 & $4.4 \%$ & $0.68[0.47,0.97]$ & $\rightarrow$ & & \\
\hline Su2015 & 22 & 38 & 40 & 40 & $10.9 \%$ & $0.58[0.45,0.77]$ & - & & \\
\hline Wang et al2014 & 20 & 35 & 35 & 35 & $9.8 \%$ & $0.58[0.43,0.77]$ & - & & \\
\hline Subtotal $(95 \% \mathrm{Cl})$ & & 88 & & 91 & $25.1 \%$ & $0.60[0.50,0.71]$ & $\bullet$ & & \\
\hline Total events & 52 & & 91 & & & & & & \\
\hline \multirow{2}{*}{\multicolumn{10}{|c|}{$\begin{array}{l}\text { Heterogeneity: } \mathrm{Chi}^{2}=0.52, \mathrm{df}=2(\mathrm{P}=0.77) ; \mathrm{I}^{2}=0 \% \\
\text { Test for overall effect: } Z=5.80(P<0.00001)\end{array}$}} \\
\hline & & & & & & & & & \\
\hline Total $(95 \% \mathrm{Cl})$ & & 641 & & 652 & $100.0 \%$ & $1.31[1.21,1.42]$ & & 1 & \\
\hline Total events & 475 & & 363 & & & & & & \\
\hline $\begin{array}{l}\text { Heterogeneity: Chi } \\
\text { Test for overall effec }\end{array}$ & $\begin{array}{l}99.20, \text { df } \\
Z=6.40\end{array}$ & $\begin{array}{l}16(P< \\
<0.00\end{array}$ & $\begin{array}{l}0.00001 \\
001)\end{array}$ & $; 1^{2}=8$ & & & 0.1 & 1 & 100 \\
\hline
\end{tabular}

B

Test for subaroun differences: $\mathrm{Chi}^{2}=92.01 . \mathrm{df}=3(\mathrm{P}<0.00001) . \mathrm{I}^{2}=96.7 \%$

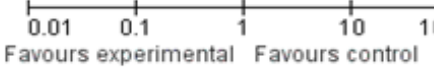

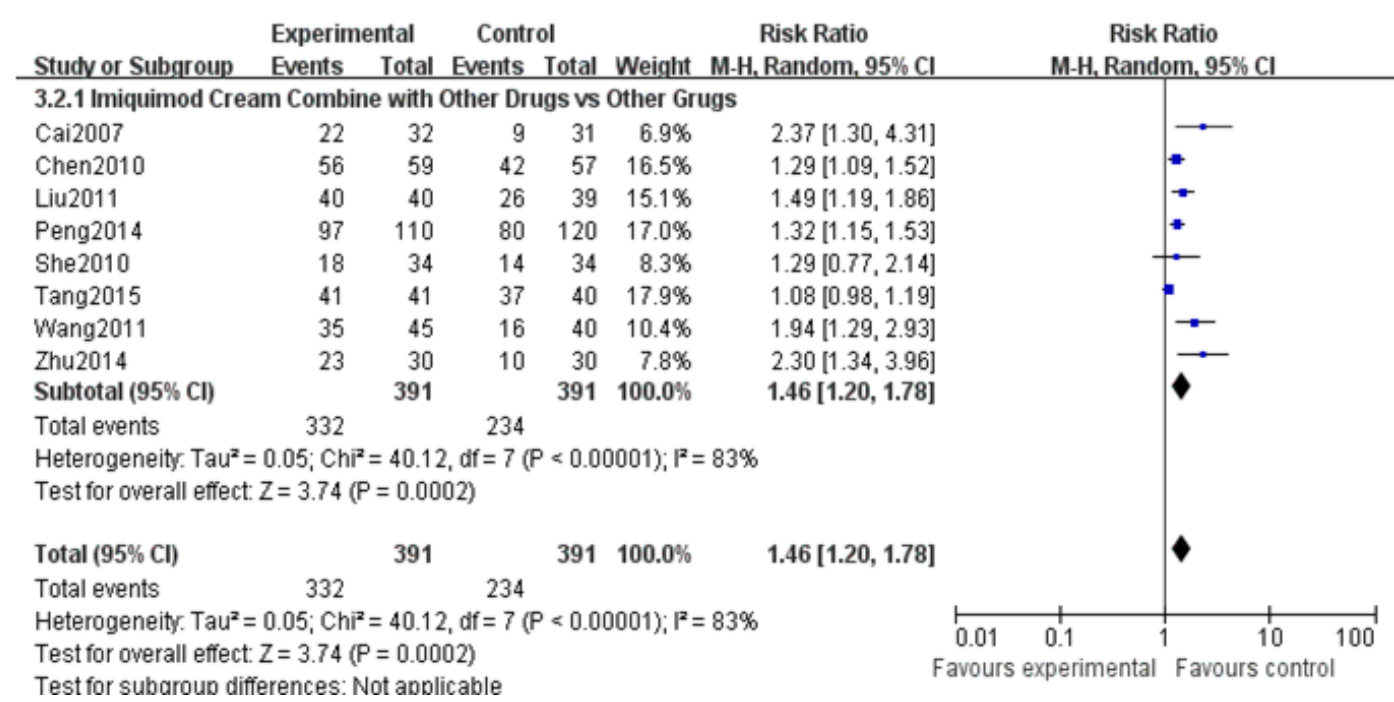

Figure 5. Forest plot for Intervention measures (5A. 3.1.1. Forest plot for comparison ofimiquimod cream therapy with tretinoin cream therapy; 5A. 3.1.2. Forest plot for comparison of imiquimod cream therapy with tazarotene gel therapy; 5A. 3.1.3. Forest plot for comparison of imiquimod cream therapy with other antiviral medications; 5A. 3.1.4. Forest plot for comparison of imiquimod cream therapy with ALA-PDT therapy; 5B. Forest plot for comparison of imiquimod cream combine with other drugs therapy with other drugs) 
Imiquimod Cream vs. Other antiviral medications: A total of 2 studies $[25,26]$ were included, of which the test group was imiquimod cream alone or combined with pidotimod powder. Pidotimod powder alone served as control group. The results showed that the effective ratein imiquimod group was higher than in control group $(\mathrm{RR}=1.71$, 95\% CI (1.29, 2.26), $\mathrm{P}=0.0002)$ (Figure 5A.3.1.3.).

Imiquimod Cream vs. ALA-PDT: A total of 3 studies [27-29] were included, of which of the test group was imiquimod cream alone once every other day and ALA-PDT in the control group. The results showed that the effective rate of imiquimod group was lower than that of ALAPDT group $(\mathrm{RR}=0.6,95 \% \mathrm{CI}(0.5,0.71), \mathrm{P}<0.00001)$ (Figure 5A.3.1.4.).

Imiquimod Cream Combined with Other Drugs vs. Others drugs: A total of 9 studies [16-24] were included, of which the test group was imiquimod cream alone or in combination with adapalene, BCG-PSN, famciclovir, transfer factor, Alpha $2 \mathrm{~b}$ interferon cream , ALA-PDT and placebo. And adapalene, BCG-PSN, famciclovir, transfer factor, Alpha 2b interferon cream and ALA-PDT were in the control groups, correspondingly. Results show that the effective rate of imiquimod (or in combination with other drugs) was higher than that of the control groups ( $\mathrm{RR}=1.46,95 \% \mathrm{CI}(1.20,1.78), \mathrm{P}=0.0002)$ (Figure $5 \mathrm{~B})$. The heterogeneity is significant due to the different intervention measures for each of the control groups.

Adverse events: 22 studies [4-17,21,23-29] reported the adverse events. However, the adverse reactions were mild, and patients could recover soon after stopping or reducing the drugs.

\section{Publication bias}

Funnel plot analysis showed that the figure was basically symmetrical upside down funnel, and there was no significant publication bias (Figure 6).

\section{Follow up}

8 studies reported the follow-up. Lium [5] followed the patients for 2 months. There was no recurrence in the cured patients in the test group, and 2 cases who significantly improved also cured. Some of the patients in the control group had increase in the lesions. Huangm [6] followed the patients for a month, test group had 2 recurrent cases, control group had 7 recurrent cases. Tang [24] closely observed the cured patients in both groups for 3 months. The recurrence cases in the test group in 1,2, 3 months were less than those in the control group, the difference was statistically significant $(\mathrm{P}<0.05)$, Wang [4] followed up for 3 months then observed both groups showed no recurrence. Su [28] followed up the cured patients for 3 months; there were no cases of recurrence. Jiang [29] followed up for half a year, the test group and the control group had no recurrence. Liu [25] followed up for 2 months the results showed no recurrence, $\mathrm{Wu}$ [26] followed up for six months, 2 recurrent cases occurred in the treatment group (5.71\%) and 4 in the control group (12.5\%).

\section{Discussion}

During the search, we found that many articles reported that imiquimod cream alone or in combination with other drugs could make a good effect on treatment of verruca planae. But there was no systematic review about efficacy and safety of imiquimod cream on the verruca planae. This systematic review included all RCT on verruca planae. The meta-analysis results showed that the effective rate of imiquimod cream in the treatment of flat wart was higher than other treatments. We found that the effective rate of ALA-PDT in treatment of verruca planae was more effective than imiquimod. However, the report about ALA-PDT use was limited.

The review made the conclusion that imiquimod was efficacy and safe on verruca planae based on the limited evidence. First, owing to the studies of imiquimod on verruca planae was investigated mostly

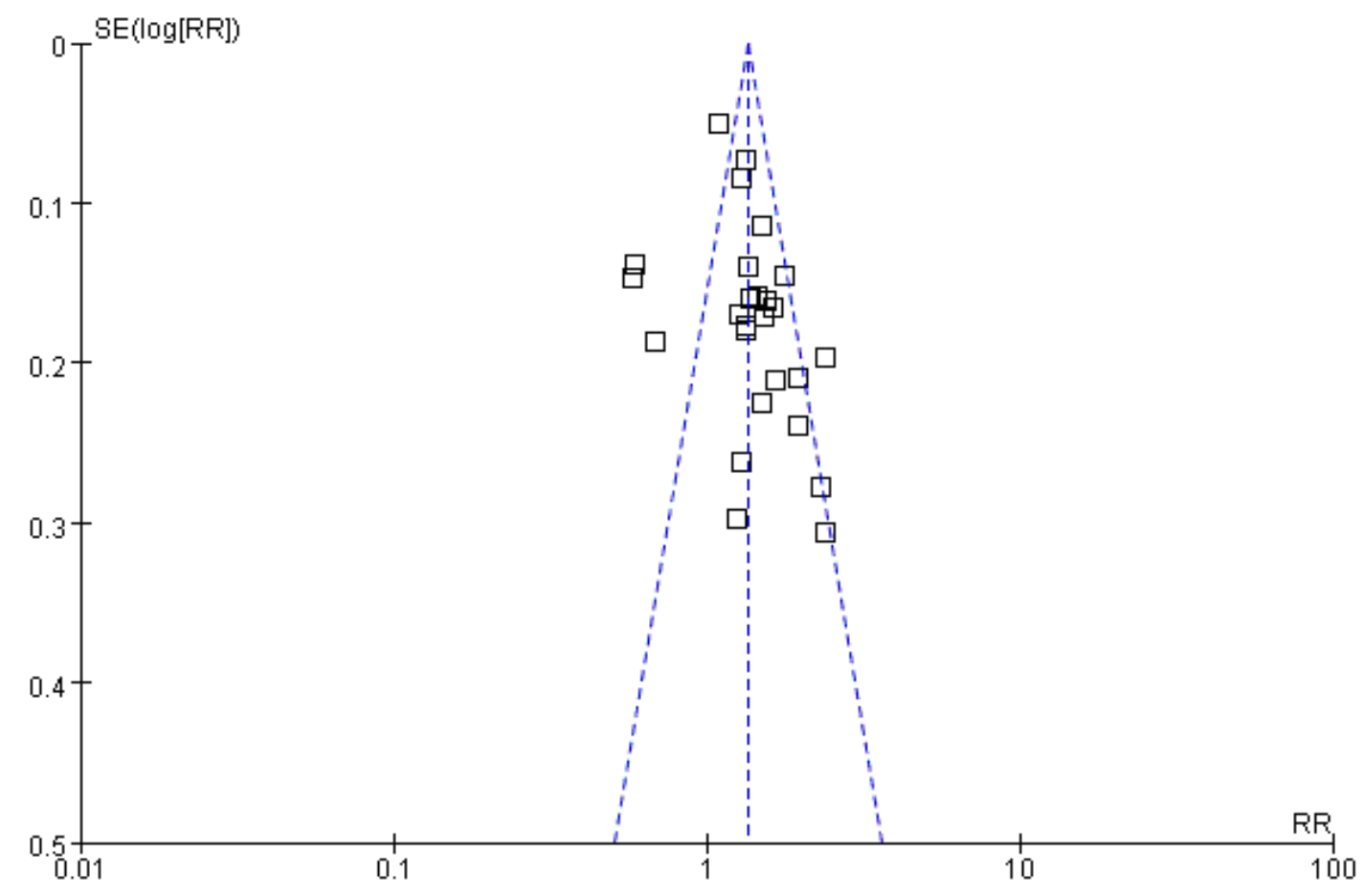

Figure 6. Funnel plot for evaluating publication bias. 
by Chinese, we included a total of 26 randomized controlled trials that all come from China which may cause language bias. Second, quality of the included studies was not high, only 1 study described exclusion or losses of follow up. All studies did not mention the blinding and allocation concealment which may cause selection bias, performance bias and measurement bias. Third, the intervention measures in the test group and the control group were different, and it was difficult to extract and combine the data. Fourth, each of the intervention measures included in the studies was relatively small. Therefore, the results of these articles need to be confirmed and re-evaluated by more RCTs with high quality, large scale and multicenteral.

\section{Acknowledgement}

We thank Yan Wu, Yan Sun, Yang Yang for their excellent technical assistance. This research is supported by Fund program: Natural Science Foundation of China (81301368); Key Laboratory of basic research foundation, Department of Education, Liaoning Provincial (LZ2014041).

\section{References}

1. James WD, Timothy GB, Dirk ME (2006) Andrew's diseases of the skin: clinical dermatology (10 ${ }^{\text {th }}$ edtn) Elsevier, Philadelphia, USA.

2. Richwald GA (1999) Imiquimod. Drugs Today 35: 497-511.[Crossref]

3. Schwab RA, Elston DM (2000) Topical imiquimod for recalcitrant facial flat warts. Cutis 65:160-162.[Crossref]

4. Wang Q (2014) Evaluating the clinical efficacy of treating Verruca Plana with 5\% Imiquimod Cream and Polysaccharide Nucleic Acid Fraction of Calmette-Guerin (BCG-PSN). Chinese Medical Cosmetology 4: 109-110.

5. Liu GX, Chen J (2014) Clinical Observation Treating Verruca Plana with $5 \%$ Imiquimod Cream and Polysaccharide Nucleic Acid Fraction of Bacillus Calmette-Guerin (BCGPSN). China Medical Abstracts (Dermatology) 31: 205-205.

6. Huang MH, Qin XF, Huang Y, Yao W (2010) Observation Treating Verruca Plana withImiquimod Cream and Polysaccharide Nucleic Acid Fraction of Bacillus CalmetteGuerin (BCG-PSN). The Chinese Journal of Dermatovenereology 24: 442-443.

7. Ruan JP, Zhu RQ, Zhu HL (2005) The Study on Topical Treatment of Verruca Plana with Imiquimod Cream andTretinoin. Journal of Gannan Medical College 25: 641-642.

8. Zuo YH, Wu YQ (2012) The Study on Topical Treatment of Verruca Plana with Imiquimod Cream and Tretinoin. China Journal of Leprosy and Skin Diseases 28: 570-570.

9. Chen GX (2008) Clinical Observation Treating Verruca Plana with 5\% Imiquimod Cream. Zhejiang Journal of Integrated Traditional Chinese and Western Medicine 18: 208-208.

10. Huang Q (2006) Clinical Observation Treating Verruca Plana with 5\% Imiquimod Cream. Journal of Chinese Physician 8: 995-995.

11. Wang X, Yang GM (2006) The Study on Topical Treatment of Verruca Plana with 5\%
Imiquimod Cream. Journal of Practical Medical Techniques 13: 3981-3981.

12. Chen JY, Wang J, Song PF (2014) 45 Cases on treatment of verruca Plana with Imiquimod and Tazarotene Gel. Jiangxi Traditional Chinese Medicine 45: 39-41.

13. Xiong B (2012) The Study on Topical Treatment of Verruca Plana with Tazarotene Cream and Imiquimod Cream. Journal of Dermatology and Venereology 34: 308-308.

14. Yang HL, Li YZ, Sun R, Zhao XD (2011) The clinical observation on treatment of verruca Plana with Imiquimod and Tazarotene Gel. Heilongjiang Medicine and Pharmacy 34: 42-43.

15. Zhang JP, Zhou ZL, Shao Q (2010) The Study on Topical Treatment of Verruca Plana with Tazarotene Cream and Imiquimod Cream. The Chinese Journal of Dermatovenereology 24: 240-240.

16. Yuan B, Chen ZJ, Wang L (2015) The Study on Topical Treatment of Verruca Plana with Adapalene Gel and 5\% Imiquimod Cream. Journal of China Prescription Drug 13: 85-86.

17. Peng J, Xie YF, Wang Q (2014) The Study on Topical Treatment of Verruca Plana with Adapalene Gel and 5\% Imiquimod Cream. Journal of Leprosy and Skin Diseases 30: 596-596.

18. Chen YP (2010) The Study on Treatment of Verruca Plana with 5\% Imiquimod Cream and Polysaccharide Nucleic Acid Fraction of Bacillus Calmette-Guerin (BCG-PSN). Journal of Dermatology and Venereology 32: 37-37.

19. She XH (2010) 68 Cases on Topical Treatment of Verruca Plana with 5\% Imiquimod Cream. Proceeding of Clinical Medicine 19: 427-428.

20. Cai CB (2007) The Study on Topical Treatment of Verruca Plana with 5\% Imiquimod Cream. Journal of Clinical Dermatology 36: 400-400.

21. Wang Z (2011) The Study on Topical Treatment of Verruca Plana with Transfer Factor and 5\% Imiquimod Cream. Journal of Medical Forum 32: 73-74

22. Zhu HP, Xin L (2014) The Study on Topical Treatment of Verruca Plana with Placenta Polypeptide Injection and Imiquimod Cream. Jilin Medical Journal 35: 7818-7818.

23. Liu JP (2011) 40 Cases on treatment of verruca Plana with Imiquimod and Interferon. Journal of Dermatology and Venereology 33: 182-182.

24. Tang B, Chang XL, He CF, Qiang D, et al. (2015)The Study on Treatment of Topical Photodynamic Therapy for Flat W arts. Anhui Medical Journal 2015: 940-942.

25. Liu F, Liu Y, Wang Q (2006) The Study on Topical Treatment of Verruca Plana with 5\% Imiquimod Cream. The Chinese Journal of Dermatovenereology 20: 654-654.

26. Wu YF, Cao P (2009) The Study on Topical Treatment of Verruca Plana with Pidotimod Powder and Imiquimod Cream. China Modern Doctor 47: 51-51.

27. Wang M, Zou Q, Ye FS, Tao XH, Shuai CD (2014) Clinical Observation of Topica Photodynamic Therapy for 35 Cases of Facial Flat Warts. Journal of Diagnosis and Therapy on Dermato-Venereology 21: 465-466.

28. Su P, Gan CB, Zhang XN (2015) The Study on Topical Treatment of Photodynamic Therapy for Facial Multiple Flat Warts. Chinese Journal of Aesthetic Medicine 24: 43-45.

29. Jiang FX, Hu B, Zhao ZL, Zhu L, Liu W, et al. (2013) Clinical Observation of Topical Photodynamic Therapy for 16 Cases of Recalcitrant Facial Flat Warts. The Chinese Journal of Dermatovenereology 27: 583-584.

Copyright: (C2016 Zhang XR. This is an open-access article distributed under the terms of the Creative Commons Attribution License, which permits unrestricted use, distribution, and reproduction in any medium, provided the original author and source are credited. 